\title{
Inhibition of penile tunica albuginea myofibroblasts activity by adipose-derived stem cells
}

\author{
HESONG JIANG ${ }^{*}$, QINGQIANG GAO* , XIAOYAN CHE, LEILEI ZHU, \\ ZHENG ZHANG, YUN CHEN and YUTIAN DAI \\ Department of Andrology, Drum Tower Hospital, Affiliated to School of Medicine, \\ Nanjing University, Nanjing, Jiangsu 210008, P.R. China
}

Received March 22, 2017; Accepted August 24, 2017

DOI: $10.3892 /$ etm.2017.5179

\begin{abstract}
The activation of tunica albuginea myofibroblasts (MFs) serves an essential role in Peyronie's disease (PD). Increasing evidence has reported that adipose tissue-derived stem cells (ADSCs) have been demonstrated to attenuate the symptoms of PD in animal models. However, the mechanisms of the antifibrotic effects of ADSCs in PD remain to be fully elucidated. In the present study, the inhibitory effects and possible mechanism of ADSCs on the activation of MFs derived from rat penile tunica albuginea were investigated. ADSCs were obtained from the paratesticular fat of Sprague Dawley rats. MFs were transformed from rat penile tunica albuginea fibroblasts through stimulation with $5 \mathrm{ng} / \mathrm{ml}$ tumor growth factor- $\beta 1$. Transwell cell cultures were adopted for co-culture of ADSCs and MFs. Western blot analysis was used to assess changes in the expression levels of $\alpha$ smooth muscle actin ( $\alpha$ SMA), collagen I, phosphorylated (p)-SMAD family member 2 (Smad2), Smad2, ras homolog family member A (RhoA), Rho associated coiled-coil containing protein kinase (ROCK)1 and ROCK2, caspase3, caspase9, and matrix metalloproteinases (MMPs). Collagen gel assays were used to assess cell contractility. Additionally, the concentration of hydroxyproline in the culture medium was detected using commercially available kits. It was demonstrated that ADSCs reduced the expression of $\alpha \mathrm{SMA}$ and collagen I of MFs. Furthermore, p-Smad2, RhoA, ROCK1 and ROCK2 expression was significantly reduced in the MFs+ADSCs group compared with that in the MFs-only culture, while the expression of MMPs (MMP2, MMP3, MMP9 and MMP13) and caspases (caspase3 and caspase9) was upregulated. In addition, ADSCs were able to
\end{abstract}

Correspondence to: Professor Yutian Dai, Department of Andrology, Drum Tower Hospital, Affiliated to School of Medicine, Nanjing University, 321 Zhong Shan Road, Nanjing, Jiangsu 210008, P.R. China

E-mail: 13913957628@163.com

*Contributed equally

Key words: adipose tissue-derived stem cells, myofibroblasts, Peyronie's disease, fibrosis downregulate the concentration of hydroxyproline in the culture medium of MFs and reverse the contraction of MFs. Collectively, these results suggested that ADSCs inhibited the activation of MFs, decreased collagen production, and suppressed the contraction of myofibroblasts, via Smad and RhoA/ROCK signaling pathways. Furthermore, ADSCs reduced the deposition of collagen and promoted the apoptosis of MFs via MMPs, and caspases. Accordingly, the application of ADSCs may provide a novel therapeutic strategy for PD.

\section{Introduction}

Peyronie's disease (PD) is a penile fibrotic disease characterized by the presence of tunical plaques which can clinically lead to penile pain, penile deformity including curvature, narrowing and hourglass, and potential erectile dysfunction (1). In almost all fibrotic diseases, one of most important mechanisms is that fibroblasts transdifferentiate into $\alpha$-smooth muscle actin ( $\alpha$ SMA)-positive myofibroblasts (2). In Peyronie's disease, tunica albuginea myofibroblasts (MFs) were mainly derived from tunica albuginea fibroblasts (TAFs) induced by TGF- $\beta$ (3). Previously, Vernet D found that $\alpha$ SMA positive myofibroblasts content was considerably increased in the human Peyronie's disease and TGF $\beta 1$-induced rat plaques as compared to control tunica albuginea by Immunohistochemistry (4). Furtherly when compared the gene expression profiling between the PD plaques and the nomal penile tunica abuginea, Magee TR that the mainly up-regulated genes were involved in the function of myofibroblasts, such as myofibroblast differentiation and collagen synthesis, while the down-regulated genes were that inhibit these processes, collagenase and matrix metalloproteinases (MMPs) $(5,6)$. On the one hand myofibroblasts could secrete extracellular matrix (ECM) components (particularly collagen), and in the other hand, MFs had the function of automatic contraction $(7,8)$. These processes could resulted in the PD plaques and the penile deformity.

MSCs were a kind of stem cells with the function of self-renewal and multi-directional differentiation. In mounting experimental or pre-clinical studies, researchers found that mesenchymal stem cells (MSCs) could prevent the development of tissue fibrosis $(9,10)$. Previously studies proved that MSCs could slow the progression of fibrosis, and reverse functional remodeling in heart, liver, kidney, and lung 
tissues (11-15). Adipose tissue-derived stem cells (ADSCs) belonged to the family of MSCs. Because of abundant adipose tissues and simplely obtained method, ADSCs were the mainly source of adult stem cells. Furthermore, compared with other stem cells, ADSCs had fewer ethical problems and lower immunogenicity (16). Therefore, there were a lot of studies involved in the roles of ADSCs in attenuating fibrotic disease, such as liver cirrhosis, idiopathic pulmonary fibrosis and kidney fibrosis (17-19). It will be highlighted that ADSCs were progressively used to recover the animal model of Peyronie's disease. The mechanisms of anti-fibrosis by ADSCs had not been completely elucidated $(20,21)$, while the paracrine signaling is considered as one of the main underlying mechanisms of the therapeutic effects of MSCs (12). Otherwise, the balance of activation vs. inactivation and proliferation vs. apoptosis of TAMFs played the important role in Peyronie's disease. Furthermore, MMPs participated in the regulation and clearance of ECM secreted by MFs, and automatic contraction of MFs was mainly involved by the RhoA/ROCK signaling pathway $(22,23)$.

Therefore, we performed the following experiments by using the transwell coculture of ADSCs and tunica albuginea MFs. Firstly, we assess whether ADSCs regulated the secretion of collagen by MFs and the contraction of MFs. Additionally, we explored the proliferation vs. apoptosis of MFs when cocultured with ADSCs. Finally, we investigated MMPs and RhoA/ROCK signaling pathway of TAMFs when cocultured with ADSCs.

\section{Materials and methods}

Cell culture. We used 3 male Sprague-Dawley rats (from the Animal Feeding Center of Nanjing Medical University, Jiangsu, China) to isolate the ADSCs and tunica albuginea fibroblasts (TAFs) In independent experiments. Under $4 \%$ chloral hydrate anesthesia, penile tissue paratesticular fat and penile tunica albuginea were harvested to isolate the ADSCs and TAFs, then all rats were euthanized. All procedures were approved by the Institutional Animal Care and Use Committee of Nanjing University.

Monoculture. ADSCs. ADSCs were isolated from paratesticular fat of SD rats and cultured as described previously (24). Rat paratesticular adipose tissues were minced and incubated with $0.1 \%$ collagenase I (Catalog No. 17100-017, Life Technologies, GIBCO) for $1 \mathrm{~h}$ at $37^{\circ} \mathrm{C}$. The digested tissues were filtered through a $75-\mu \mathrm{m}$ mesh, then centrifuged at $200 \mathrm{x} \mathrm{g}$ for $5 \mathrm{~min}$, and the cell precipitates were re-suspended in Dulbecco's modified Eagle's medium (DMEM) containing 10\% fetal bovine serum (FBS; both from Gibco, Carlsbad, CA, USA) and $1 \%$ antibiotics $(100 \mathrm{mg} / 1$ streptomycin and $100 \mathrm{U} / \mathrm{ml}$ penicillin) at $37^{\circ} \mathrm{C}$ in a humidified $95 \%$ air $/ 5 \% \mathrm{CO}_{2}$ environment. Surface markers (such as CD90, CD34, CD45, CD44) were identified by flow cytometric analysis of passage 3 ADSCs as peformed in our previous study (25). The passage 3-8 ADSCs were starved with serum-free medium with $5 \mathrm{ng} / \mathrm{ml}$ TGF- $\beta 1$ for $24 \mathrm{~h}$ and used to the following experiments.

Preparate and monoculture MFs. Primary TAFs were isolated, cultured and identified as previously described (26). Briefly,
TAFs from Sprague-Dawley rats were cultured in DMEM, supplemented with $10 \%$ FBS (both from Gibco, Carlsbad, CA, USA) and $1 \%$ antibiotics (100 mg/l streptomycin and $100 \mathrm{U} / \mathrm{ml}$ penicillin) at $37^{\circ} \mathrm{C}$ in a humidified $95 \%$ air $/ 5 \% \mathrm{CO} 2$ environment. The TAFs were then treated with TGF- $\beta 1(10 \mathrm{ng} / \mathrm{ml}$; Sigma) for $24 \mathrm{~h}$. As described in our previous study, TAFs were transformated into myofibroblasts which had high expression of aSMA (3). Then myofibroblasts were maintained with $5 \mathrm{ng} / \mathrm{ml}$ TGF- $\beta 1$ for following experiments.

Transwell cocluture of MFs and ADSCs. The transwell coculture delivered a great environment that both types of cells shared culture medium but did not directly contact (27). Cells were digested with trypsin, re-suspended in a serum-free DMEM medium with $5 \mathrm{ng} / \mathrm{ml}$ TGF- $\beta 1$. ADSCs were plated into the matrigel coated Transwell chambers (Corning, NY, USA) and MFs were planted in 12-well plates. Then Chambers were inserted into 12-well plates filled with serum-free DMEM medium with $5 \mathrm{ng} / \mathrm{ml}$ TGF- $\beta 1$. The system was incubated at the temperature of $37^{\circ} \mathrm{C}$ for 24,48 or $72 \mathrm{~h}$ of incubation (Fig. 1A).

Measurement of hydroxyproline concentration. Hydroxyproline was used to estimate the secretion of total collagen in the medium according to the method described by Woessner (28) and the protocol included in the hydroxyproline kit (A030-1; Nanjing Jiancheng Bioengineering Institute, Jiangsu, China). Duplicate 300- $\mu$ l aliquots of medium were taken from each sample and transferred to microtiter plates, and the absorbance of each was determined at $550 \mathrm{~nm}$ by spectrophotometry. The hydroxyproline concentrations of the sample pending to be tested were calculated using a linear standard curve and are presented as $\mu \mathrm{g} / \mathrm{ml}$ medium.

Collagen gel contraction assay. All experimental operation according to the protocol (3), Collagen gels were prepared using $2 \mathrm{mg} / \mathrm{ml}$ of rat tail collagen I (Wobio, Nanjing, China) that was neutralized with $1 \mathrm{M} \mathrm{NaOH}$ and supplemented with DMEM. After cocultured with ADSCs for 24, 48 or $72 \mathrm{~h}$, MFs were digested with trypsin, re-suspended and seeded at a density of $3 \times 105$ cells $/ \mathrm{ml}$ in microtiter plates that were lubricated with FBS. Following lubrication, $0.5 \mathrm{ml}$ of the final collagen gel was incubated at $37^{\circ} \mathrm{C}$ in a humidified $95 \%$ air $/ 5 \%$ $\mathrm{CO} 2$ environment for $24 \mathrm{~h}$. Images were acquired using an Odyssey Scanning System (LI-COR Biosciences, Lincoln, NE, USA), and the surface areas were quantified using ImageJ software (NIH, Bethesda, MD, USA).

Western blot analysis. Cells were harvested at scheduled time and washed in phosphate-buffered saline (PBS) and then lysed in RIPA buffer (Sigma). Total protein concentrations were measured using Bicinchoninic acid (BCA) reagent (Beyotime Biotech, Jiangsu, China) were used to determine the protein concentration. Western blot was performed as previously described (29). Briefly, Proteins were separated in $10 \%$ sodium dodecyl sulfate-polyacrylamide gel electrophoresis (SDS-PAGE) and were then electrophoretically transferred onto polyvinylidene fluoride (PVDF) membranes at $200 \mathrm{~mA}$ for 60-120 $\mathrm{min}$. The membranes were blocked 5\% non-fat milk in TBST at $37^{\circ} \mathrm{C}$ for $1 \mathrm{~h}$. Then membranes were incubated 
A

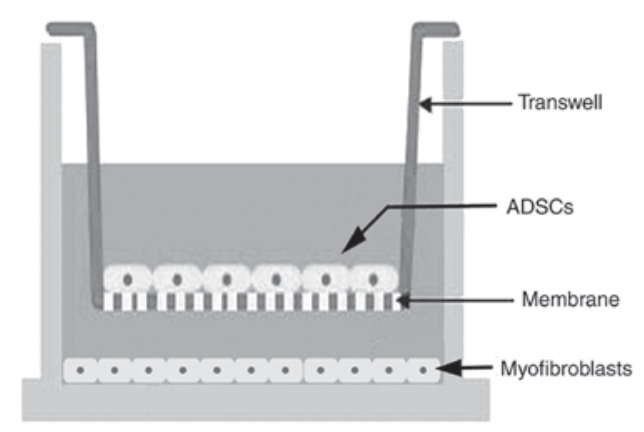

C

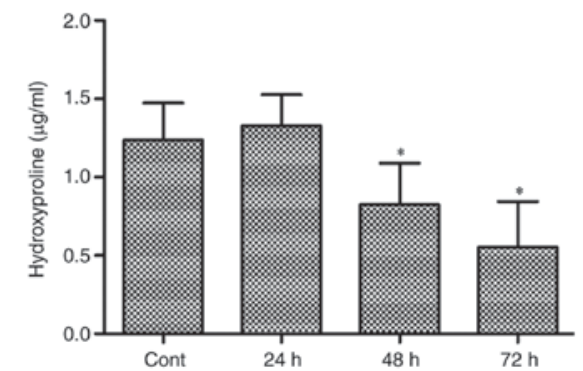

B
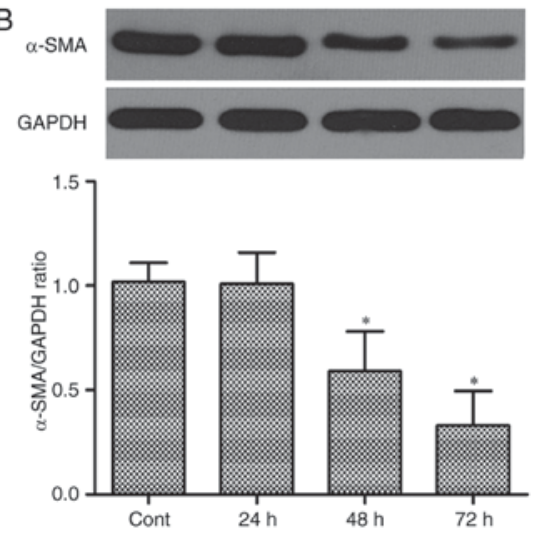

D
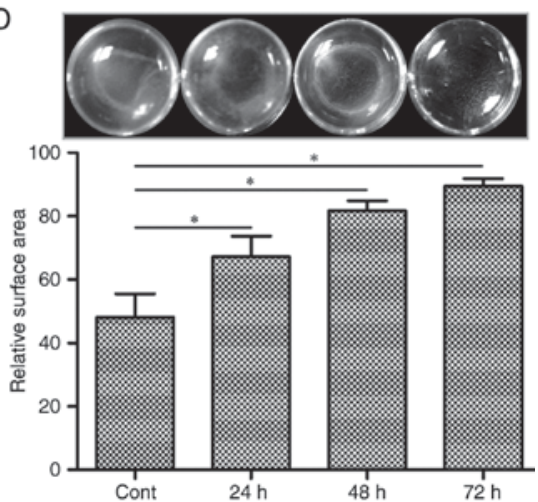

Figure 1. Adipose tissue-derived stem cells (ADSCs) attenuate the activation of myofbiroblasts (MFs). (A) Illustration of Transwell cell co-cultures of ADSCs and MFs. (B) ADSCs inhibit the expression of $\alpha$-smooth muscle actin ( $\alpha$ SMA) in MFs. Representative western blots showing the total protein levels of $\alpha$ SMA in a time dependent manner. The relative levels of $\alpha$ SMA to GAPDH are indicated by the corresponding bar chart. Cont: the expression of $\alpha$ SMA in monocultured MFs with $5 \mathrm{ng} / \mathrm{ml}$ TGF- $\beta 1$ for $72 \mathrm{~h}$. (C) ADSCs reduce the concentration of hydroxyproline in culture medium with $5 \mathrm{ng} / \mathrm{ml}$ TGF- $\beta 1$ of MFs in a time dependent manner. Cont: The concentration of hydroxyproline in culture medium of monocultured MFs with $5 \mathrm{ng} / \mathrm{ml} \mathrm{TGF}-\beta 1 \mathrm{for} 72 \mathrm{~h}$. (D) Effects of ADSCs on MF contraction. MFs were co-cultured with ADSCs for 24,48 and $72 \mathrm{~h}$, then MFs seeded in $2.5 \mathrm{mg} / \mathrm{ml}$ of rat tail collagen with $5 \mathrm{ng} / \mathrm{ml} \mathrm{TGF}-\beta 1 \mathrm{for} 24 \mathrm{~h}$. ADSCs reversed spontaneous collagen gel contraction and also increased collagen gel surface area compared with controls (Cont) in a time dependent manner. Cont: MFs monocultured with $5 \mathrm{ng} / \mathrm{ml}$ TGF- $\beta 1$ for $72 \mathrm{~h}$, then seeded in collagen gels. Quantitative data are presented as the means \pm SD. Three independent experiments were performed. ${ }^{*} \mathrm{P}<0.01$ vs. Cont.

at $4^{\circ} \mathrm{C}$ overnight with the primary antibodies, [anti- $\alpha \mathrm{SMA}$ (Rabbit, 1:1,000, Sigma), anti-Smad2 (Rabbit, 1:1,000, Sigma), anti-phosphorylated (p-)Smad2 (Rabbit, 1:1,000, Sigma), anti-RhoA (Mouset, 1:1,000, Sigma), anti-ROCK1 (Rabbit, 1:1,000, Sigma), anti-ROCK2 (Rabbit, 1:1,000, Sigma), anti-Col1 (Rabbit, 1:1,000; Abcam), MMP-2 (Rabbit, 1:1,000; CST), MMP-3 (Rabbit, 1:1,000; CST), MMP-9 (Rabbit, 1:1,000; CST), MMP-13 (Rabbit, 1:1,000; Abcam), anti-GAPDH (Mouse, 1:1,000, Sigma)]. After washing the membranes with TBST, the membranes were then incubated with the corresponding secondary antibody (horseradish peroxidase conjugated goat anti-rabbit/mouse IgG; 1:10,000, Wuhan Boster Biological Technology Ltd., Wuhan, China) at room temperature for $2 \mathrm{~h}$. The immunoreactive traces of membranes were detected by chemiluminescence (ECL) Kit (Beyotime Biotech) and an Odyssey Scanning System (LI-COR Biosciences). Finally, ImageJ software was used to quantify the expression levels of the target proteins by calculating the ratio of the mean intensity of each target protein to GAPDH.

Statistical analysis. All data were obtained from at least 3 individual experiments and expressed as the mean values \pm standard deviation (SD). Statistical analysis was performed by Student's t-tests using SPSS 16.0 software. A P-value $<0.05$ was considered to a statistically significant.

\section{Results}

ADSCs attenuated the expression of $\alpha$-SMA in tunica albuginea $M F s$. Since the hallmarker of activated myofibroblasts is aSMA, western blots were performed to analyse the effects of ADSCs on the $\alpha$ SMA protein levels of tunical abluginea MFs. Compared to monocultured MFs in the presence of TGF- $\beta 1$ (5 $\mathrm{ng} / \mathrm{ml}$ ), the $\alpha$ SMA protein level of MFs was reduced when transwell coculture with ADSCs in a time-dependent manner in the presence of TGF- $\beta 1$ (5 ng/ml; Fig. 1B).

ADSCs could reduces the levels of hydroxyproline in the culture mediam of MFs. Hydroxyproline was an general amino acid which was specificly expressed in collagen proteins. Therefore hydroxyproline was often used to measure secretion and deposition of collagen protein (28). As shown in Fig. 1C, cocultured with ADSCs, the levels of hydroxyproline was suppressed in the culture medium of MFs (CM-MFs) when compared with monocultured MFs, although TGF- $\beta 1(5 \mathrm{ng} / \mathrm{ml})$ was existed in MFs with or 

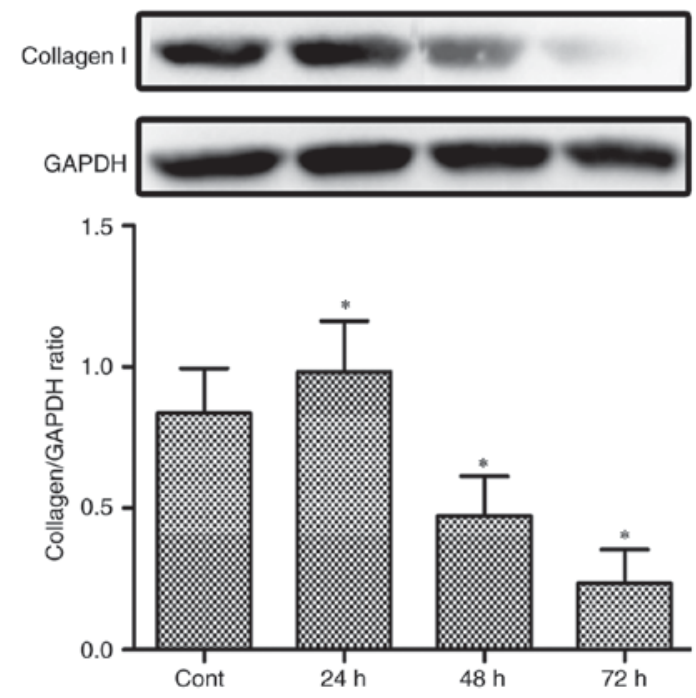

Figure 2. Adipose tissue-derived stem cells (ADSCs) inhibit the expression of Collagen I in myfibroblasts (MFs). Representative western blots showing the total protein levels of Collagen I in a time dependent manner. The relative levels of Collagen I to GAPDH are indicated by the corresponding bar chart. Cont: The expression of Collagen I in monocultured MFs with $5 \mathrm{ng} / \mathrm{ml}$ TGF- $\beta 1$ for $72 \mathrm{~h}$. Quantitative data are presented as the means \pm SD. Three independent experiments were performed. ${ }^{*} \mathrm{P}<0.01$ vs. Cont.

without ADSCs $(\mathrm{P}<0.01)$. Otherwise, the attenuate effect of hydroxyproline in CM-MFs was also in a time-dependent manner (Fig. 1C).

The collagen gel contraction inducing by MFs is suppressed when cocultured with ADSCs. Collagen gel contraction assay was carried out to analyze the inhibitory effects of ADSCs on the contractile process of MFs. The MFs mixed with $5 \mathrm{ng} / \mathrm{ml}$ TGF- $\beta 1$ were applied to collagen gels and incubated with or without culture mediam of ADSCs (CM-ADSCs) for 24,48 and $72 \mathrm{~h}$. MFs could induce contraction of collagen gel as shown in control wells. Compared to control wells, CM-ADSCs significantly suppressed gel contraction in a time-dependent manner $(\mathrm{P}<0.01)$. Futhermore, CM-ADSCs could almost completely reversed this contraction at $72 \mathrm{~h}$ (Fig. 1D).

ADSCs attenuated the activation of Smad signaling pathway in MFs. The Smad signaling pathway is a classical TGF- $\beta 1$ signaling pathway, which promotes transcription, translation, and synthesis of collagen. Smad2 is the key factor in the TGF- $\beta 1 /$ Smad signaling pathway (30). In this study, collagen I expression was inhibited in MFs co-cultured with ADSCs compared with in monocultured MFs at $48 \mathrm{~h}$ and 72 h (Fig. 2). Furthermore, compared with monocultured MFs, p-Smad2, the activated state of Smad 2, were down regulated in MFs co-cultured with ADSCs, although TGF- $\beta 1$ $(5 \mathrm{ng} / \mathrm{ml})$ was supplemented. Additionally, no significant differences in total Smad2 protein levels were observed between MFs co-cultured with ADSCs and monocultured MFs (Fig. 3).

ADSCs could promote the expression of matrix metalloproteinases (MMPs) in MFs. One of the functions of MMPs was to degradate collagen fibers. Therefore, MMP-2, $-3,-9$, and -13 were determined by western blot from monocultured MFs, monocultured ADSCs or co-cultures of MFs with ADSCs.
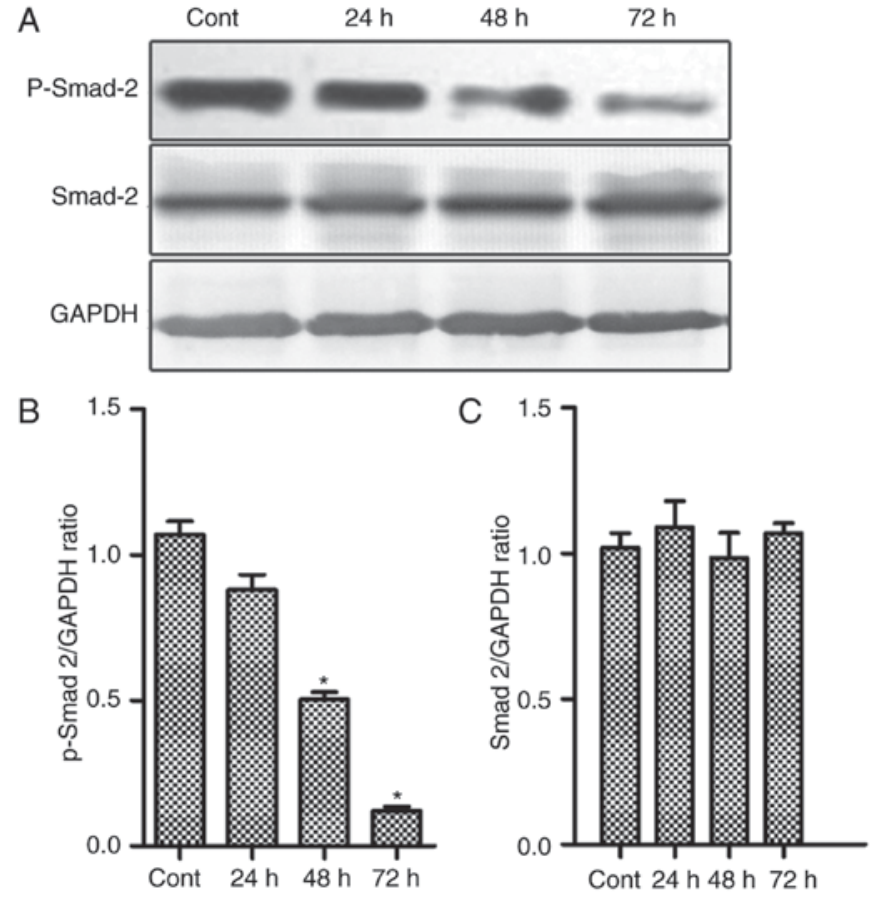

Figure 3. Effects of adipose tissue-derived stem cells (ADSCs) on activation of Smad-dependent signaling pathways. (A) Representative western blots showing the phosphorylation and total protein levels of Smad2 in tunica albuginea derived myofibroblasts (MFs) in a time dependent manner. (B and C) The relative levels of $\mathrm{p}-\mathrm{Smad} 2$ or Smad2 to GAPDH are indicated by the corresponding bar chart. Cont: The expression of p-Smad2 or Smad2 in monocultured MFs with $5 \mathrm{ng} / \mathrm{ml}$ TGF- $\beta 1$ for $72 \mathrm{~h}$. Three independent experiments were performed. $\mathrm{P}<0.01$ vs. Cont.

Compared with monocultured MFs or ADSCs, the expressions of MMP-2, $-3,-9$, and -13 were markedly increased in the presence of MFs coclutured with ADSCs (Fig. 4).

Activation of the Rho/ROCK pathway by MFs was inhibited via cocultured with ADSCs. The Rho/ROCK signaling pathway also play a major role in fibrotic disease by promoting cell contraction and migration (24-26). To elucidate the molecular mechanisms of the MFs contraction, we thus performed an in vitro experiment in which the cells were pre-treated with TGF- $\beta 1(5 \mathrm{ng} / \mathrm{ml})$ for $24 \mathrm{~h}$ and then with or without ADSCs cocultured. Compared to the monocultured MFs, the expression of RhoA was significantly lower after $72 \mathrm{~h}$ in MFs cocultured with ADSCs, and the expression of ROCK1 or ROCK2, which were the receptor of RhoA, were slightly decreased when cocultured with ADSCs (Fig. 5).

ADSCs could promote apoptosis of MFs by increasing expression or activation of caspase 3 and caspase9. Most members of caspase family play a important role in apoptosis and were often considered as the protein hallmarks of cell apoptosis. Caspases associated apoptosis are subcategorised as initiator caspases (eg.caspase9) and executioner caspases (eg. caspase 3). In the present study, we investigated whether ADSCs were able to regulate the expression of caspase 3 and caspase 9 in MFs. Compared to monocultured MFs, the expression of caspase 3 and caspase 9 of MFs were significantly elevated when cocultured with ADSCs. A similar differential effect of cleaved-caspase 3 and cleaved-caspase 9 were also seen 
A

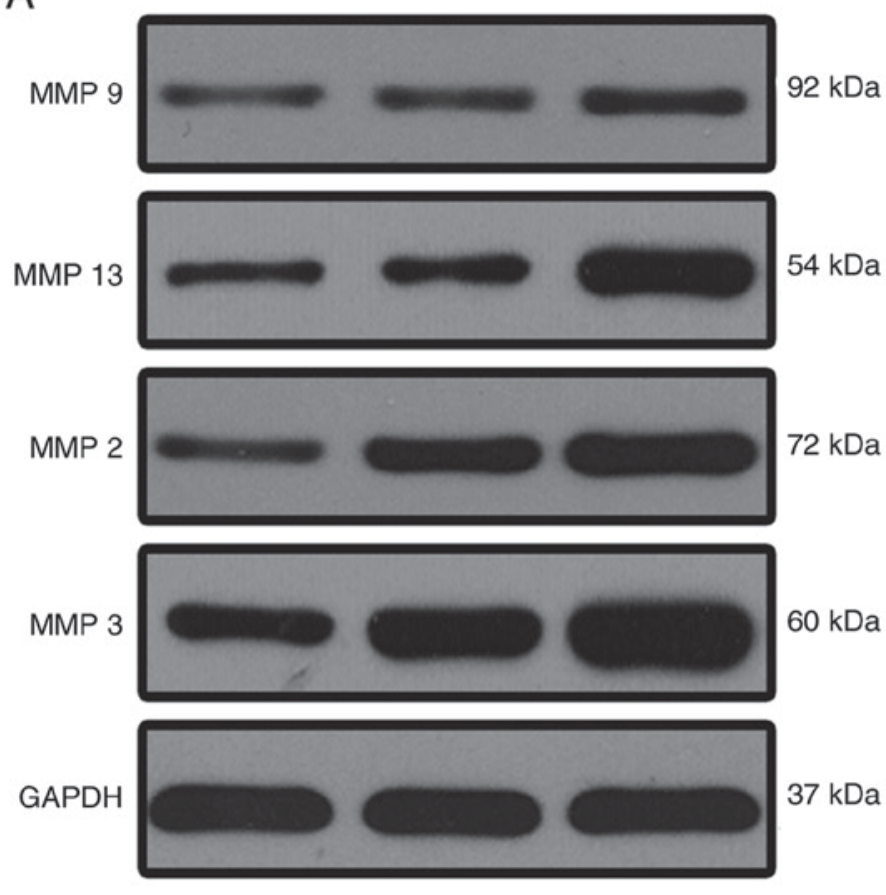

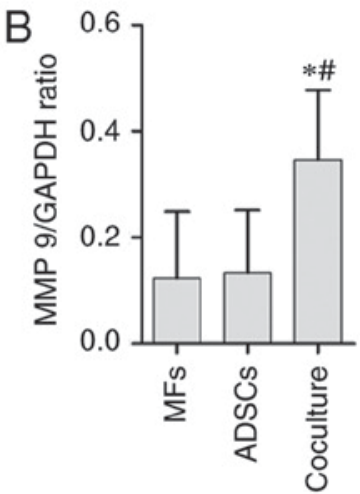
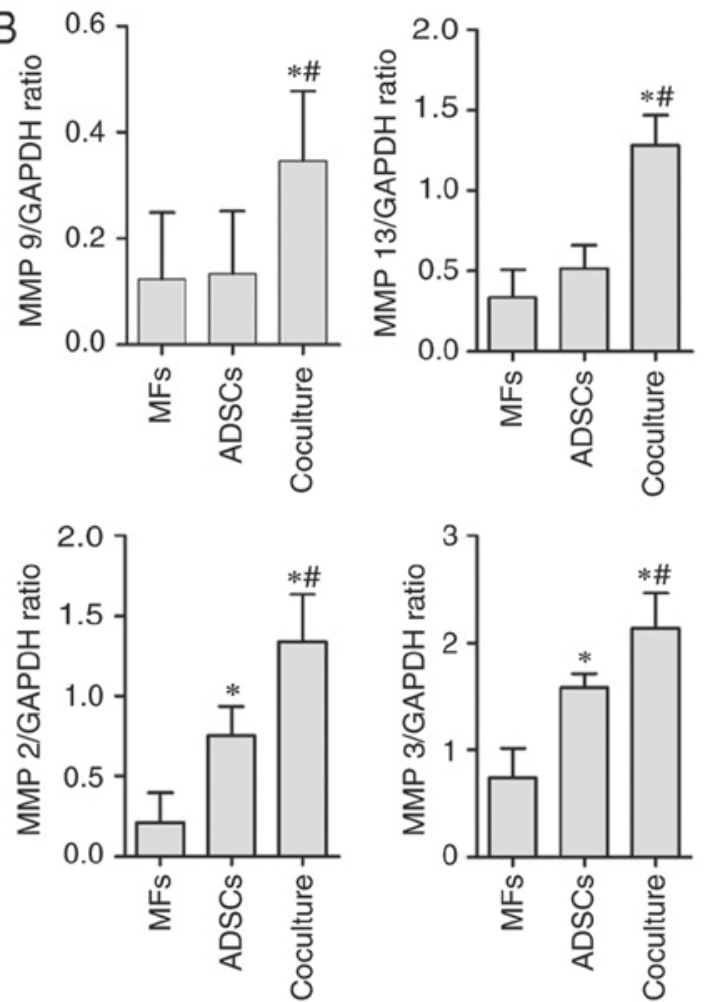

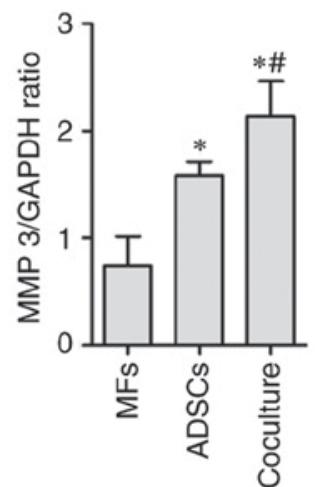

Figure 4. Expression of MMP-2, -3, -9, and -13, following co-culture of myofibroblasts with adipose tissue-derived stem cells (ADSCs) in $5 \mathrm{ng} / \mathrm{ml}$ TGF- $\beta 1$ CM for $72 \mathrm{~h}$. (A) Representative western blots showing the protein levels of MMP-2, -3, -9 and -13 in MFs by monoculture or co-culture with ADSCs, and monocultured ADSCs, respectively. (B) The relative levels of MMP-2, -3, -9 and -13 to GAPDH are indicated by the corresponding bar chart. Comparisons are made with monocultures of MFs and ADSCs in $5 \mathrm{ng} / \mathrm{ml}$ TGF- $\beta 1$ for $72 \mathrm{~h}$. Data are presented as means \pm SD. Three independent experiments were performed. ${ }^{*} \mathrm{P}<0.01$, vs. monocultured MFs; ${ }^{\mathrm{P}} \mathrm{P}<0.01$, vs. monocultured ADSCs. MMP, matrox mettaloproteinase; $\mathrm{CM}$, culture medium.

A
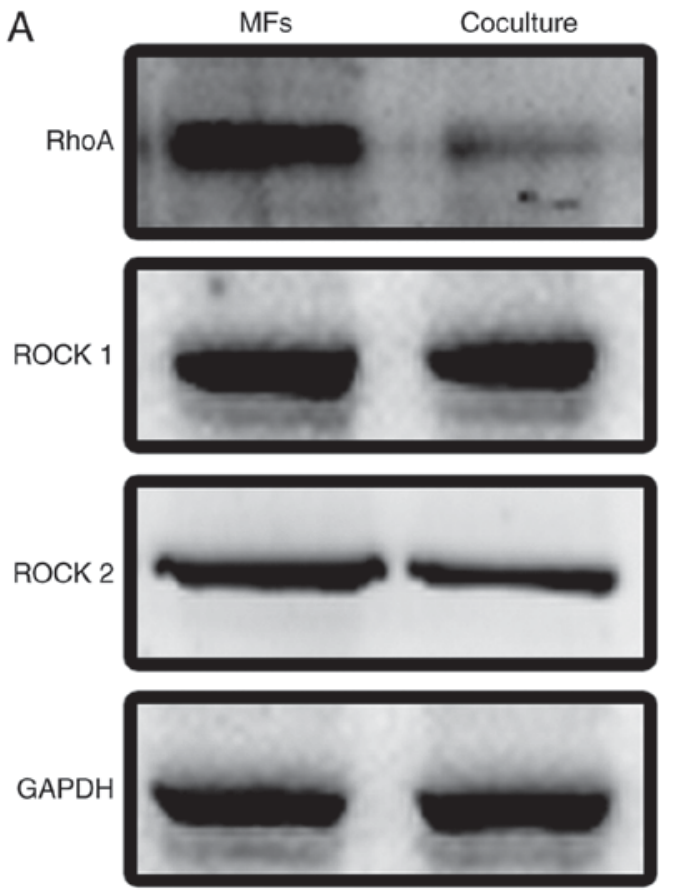

B
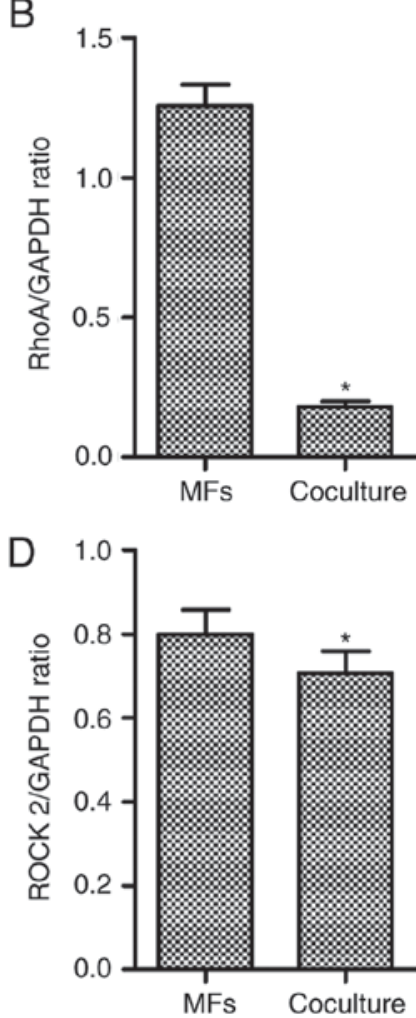

C

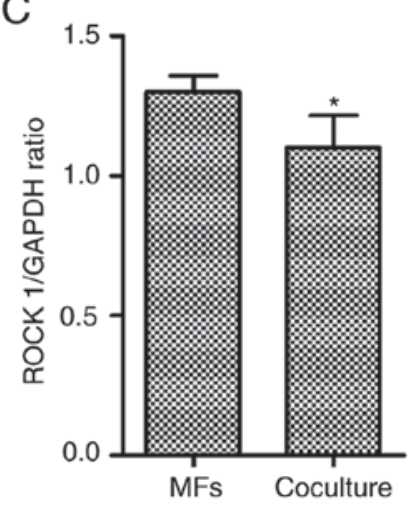

Figure 5. Effects of adipose tissue-derived stem cells (ADSCs) on activation of Smad-independent signaling pathways. (A) Representative western blots showing the total protein levels of RhoA, ROCK1 or ROCK2 in tunica albuginea derived myofibroblasts (MFs), which were co-cultured with or without ADSCs for $72 \mathrm{~h}$. (B-D) The relative levels of RhoA, ROCK1 or ROCK2 to GAPDH are indicated by the corresponding bar chart. Cont: The expression of RhoA, ROCK1 or ROCK 2 in monocultured MFs with $5 \mathrm{ng} / \mathrm{ml}$ TGF- $\beta 1$ for $72 \mathrm{~h}$. Three independent experiments were performed. "P<0.01 vs. monocultured MFs. 
A
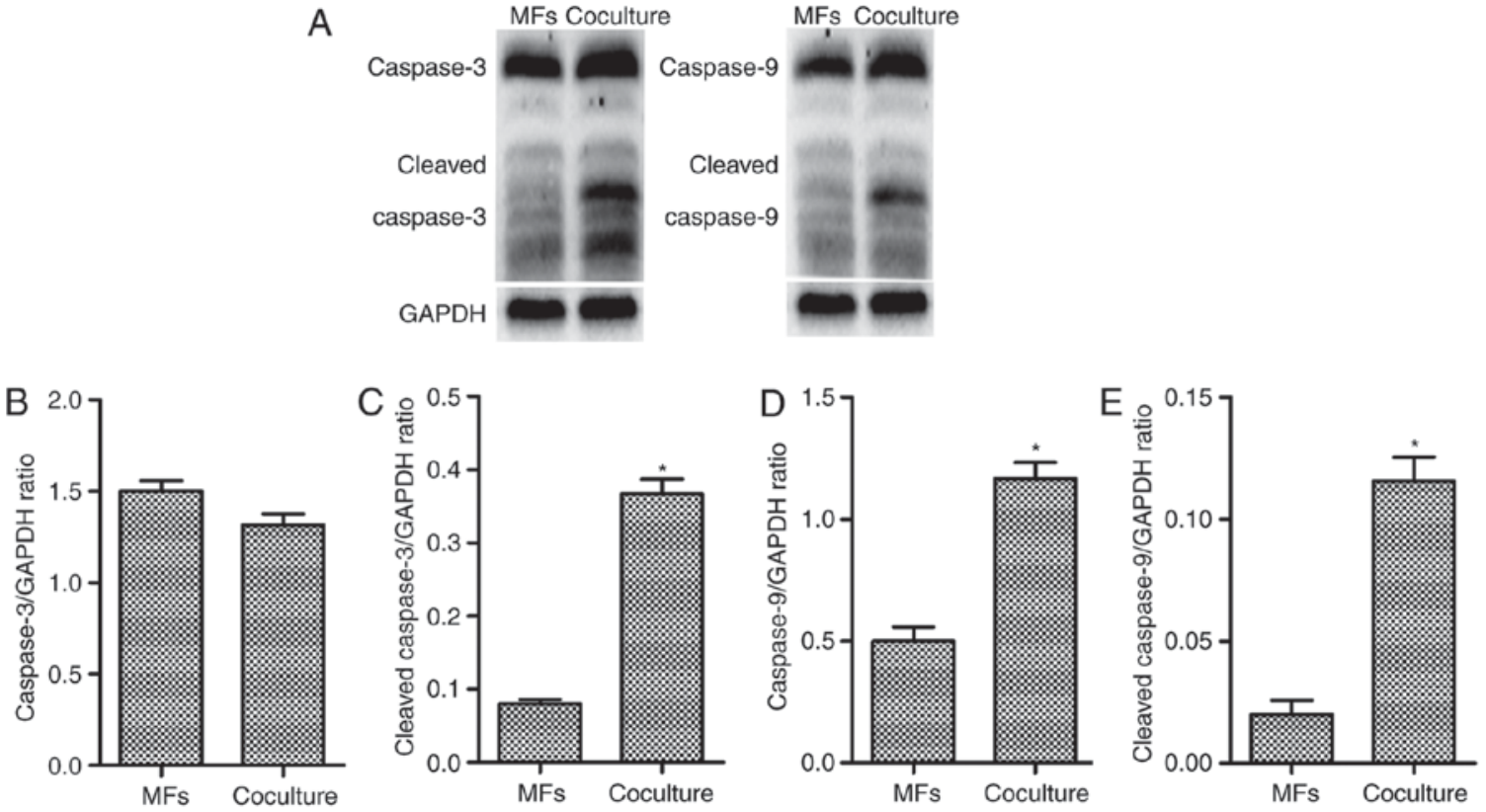

Figure 6. Adipose tissue-derived stem cells (ADSCs) could increased the expression of apoptosis-related proteinon in tunica albuginea derived myofibroblasts (MFs). (A) Representative western blots showing the total protein levels of caspase 3 or caspase9 in MFs, which were co-cultured with or without ADSCs for $72 \mathrm{~h}$. (B-E) The relative levels of caspase 3 or caspase 9 and cleaved caspase 3 or cleaved caspase 9 to GAPDH are indicated by the corresponding bar chart. Cont: The expression of caspase 3 or caspase 9 in monocultured MFs with $5 \mathrm{ng} / \mathrm{ml}$ TGF- $\beta 1$ for $72 \mathrm{~h}$. Three independent experiments were performed. "P $<0.01$ vs. monocultured MFs.

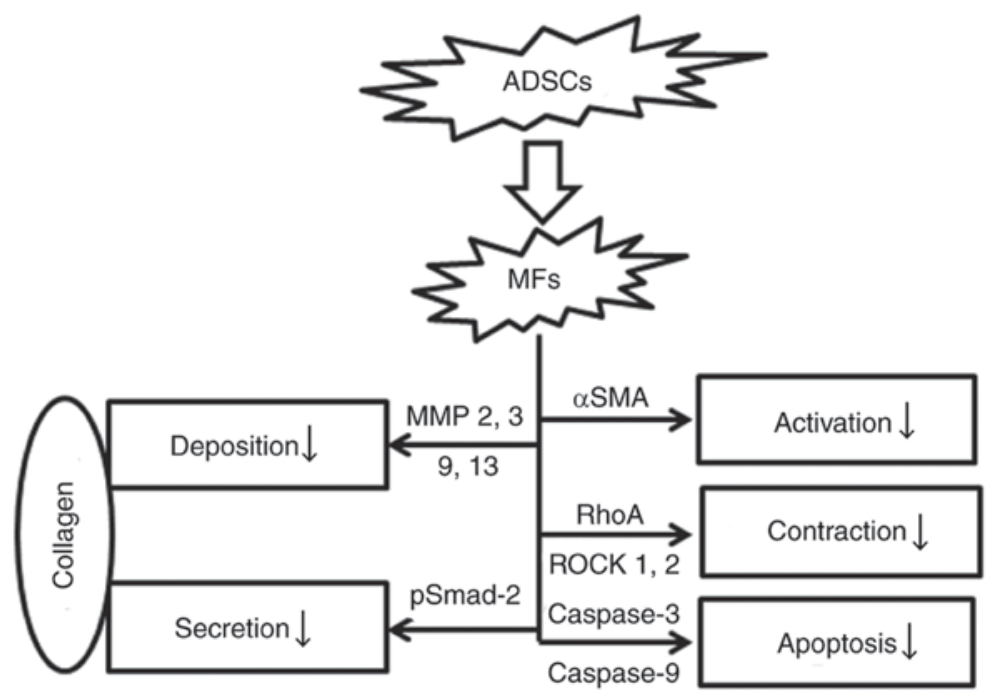

Figure 7. Proposed model of Adipose tissue-derived stem cells (ADSCs) inhibit the activation of tunica albuginea derived myofbiroblasts (MFs).

in co-cultures of MFs with ADSCs or monocultured MFs (Fig. 6).

\section{Discussion}

As a kind of fibrotic dieases, the pathophysiologic process of Peyronie's disease (PD) was that the normal architecture of penile TA was damaged by repeated micro-truama and replaced by a large number of redundant ECM. ADSCs derived from rat paratesticular fat exhibited fibroblastic and spindle-shaped morphology, and expressed typical mesenchymal markers such as CD29, CD44, CD73, and CD90, and negtively expression of hematopoietic hullmarks CD45 and CD34 (25,31).
As we all known, collagen was the main component of ECM. In this study, we identified that ADSCs could exerted an antifibrotic function via reducing collagen secretion and promoting collagen degradation. Consistent with our results, Harn $\mathrm{H}$ et al found that ADSCs could reduce the expresion of $\alpha$-smooth muscle actin ( $\alpha \mathrm{SMA})$, which was the marker of hepatic stellate cells, a precursor of MFs. Further, ADSCs could inhibit the production of collagen fiber. Otherwise, ADSCs could degrade collagen fiber by increasing the expression of matrix metalloproteinase-9. Therefore, ADSCs could abrogate chemical-induced liver fibrosis (32). Moreover, after ADSC or CM-ADSCs injected to scars, Zhang Q confirmed that the regular collagen architecture was recovered and 
that the expression of $\alpha$ SMA and collagen type I were also decreased by histomorphometric and real-time quantitative polymerase chain reaction analysis (33).

Furthermore, Our results indicated that ADSCs had the ability of synthesis and secretion of MMPs, including MMP2, 3, 9, 13, whose major function was the degradation of collagen. In normal physiological process, the component of ECM was regulated by MMPs and its inhibitor, TIMP. In addition, we also found that ADSCs could elevate the expression of MMPs in myofibroblasts. Previously, Hattori et al presented that MMP-2, 3, 9, 13 were detected at higher levels in media from co-cultures with ADSCs and inflammatory cells when compared with monocultured inflammatory cells (34). Moreover, Yu et al found that ADSCs could increase the expression of MMP2 and MMP9, while down-regulated the expression of TIMP-1, meanwhile decrease the $\alpha$ SMA expression was in pancreatic stellate cells (27). A particularly important factor in the mechanism of matrix accumulation is the transforming growth factor-b (TGF-b), whose production is highly induced in many fibrotic diseases, including atherosclerosis and fibrosis of the kidney, liver and lung (35-37). Overexpressed TGF-b is proposed to stimulate fibroblast proliferation, matrix production and granulation tissue formation by TGF-b/Smad signaling pathway (38). In our study, ADSCs could reduced transnuclear expression of phospho-Smad2, which was the crucial step for initiation of TGF-b signal transduction. These results indicated that ADSCs could decrease synthetise of collagen via TGF-b/Smad signaling pathway.

RhoA is a small guanosine triphosphate (GTP) ase, belong to a member of the Ras homolog gene family. The mainly function of Rho GTPase was to regulate the actin cytoskeleton. Previous studies have suggested that RhoA and its downstream receptors, ROCK (including ROCK1 and ROCK2), were one of key signaling plathway in numerous fibrotic diseases (39). $\mathrm{Ca} 2+$ sensitization and myosin light chain (MLC) phosphorylation were induced by the progress of Rho GTPase transform to Rho GDPase, which initiated the contraction of various cells (40). In our previous study, we found that the contractility of MFs was involved in the activation of RhoA/ROCK pathway. By contrast, in current study, ADSCs could clearly attenuate the expression of RhoA, and its ROCK1 and ROCK2 in MFs. This discovery indicates that ADSCs had the ability to inhibit the contraction of MFs by modulating the RhoA/ROCK signaling pathway. Moreover, as reported in the literature, the relaxation of smooth muscle in the penis results from the inbibition of RhoA/ROCK pathway (41).

Myofibroblasts was the most important cell in PD plaque and was derived from transformation of penile TAFs, which was the largest class of cells that make up the normal TA (42). The persistence activation of myofibroblasts facilitated the progress of fibrosis, which led the penile structural remodeling. The expression of $\alpha$ SMA was the mark of activated myofibroblasts. Therefore, preventing myofibroblast activation is a potential therapeutic strategy to PD (43). In this study, we employed western blot analysis and found that ADSCs inhibited tunica albuginea MFs activation, which was indicated by a time-dependent decrease in $\alpha$ SMA expression. Apoptosis induced by loss of adhesion or adhesion-mediated signalling (termed 'anoikis') is a main pathway of tunica albuginea MFs clearance (44). Although the precise mechanism of myofibroblast apoptosis in the resolution of wound healing and tissue repair are not well defined, anoikis is likely a relevant model to study apoptotic mechanisms since cell adhesion and biomechanical tension unloading appear to play important roles in this physiological (45). In this study, ADSCs could inhibit the expression of caspase 3 (cleaved caspase 3 ) and caspase 9 (cleaved caspase9) which were the markers of cell apotosis. Previous study was undertaken to determine the regulatory mechanisms of TGF- $\beta 1$ to induce anoikis-resistance in any cell type by PI3K-AKT pathway (46). Otherwise, the apoptosis of myofibroblasts was induced by growing factor, such as HGF, whereas molecular mechanisms had yet to be fully understood (47).

In summary, we had shown that ADSCs could inhibit the activation of tunical albuginea MFs, reduce the expression of collagen in MFs through TGF- $\beta 1-$ Smad signaling pathway, suppress the contraction of myofibroblasts by RhoA-ROCK signaling pathway. In the other hand, ADSCs could degrade collagen by paracrine or promoting MFs autocrine MMPs. Furthermore, ADSCs could induce apoptosis of MFs, though the detail molecular mechanism was confusing. These findings suggested that ADSCs attenuated the development of PD (Fig. 7). The anti-fibrotic ability of ADSCs will be considered in future treatment approach of PD.

\section{Acknowledgements}

The present study was supported by grants from the National Natural Science Foundation of China (no. 81671452).

\section{References}

1. Sherer BA and Levine LA: Contemporary review of treatment options for Peyronie's disease. Urology 95: 16-24, 2016.

2. Wynn TA and Ramalingam TR: Mechanisms of fibrosis: Therapeutic translation for fibrotic disease. Nat Med 18: 1028-1040, 2012

3. Jiang HS, Zhu LL, Zhang Z, Chen H, Chen Y and Dai YT: Estradiol attenuates the TGF- $\beta 1$-induced conversion of primary TAFs into myofibroblasts and inhibits collagen production and myofibroblast contraction by modulating the Smad and Rho/ROCK signaling pathways. Int J Mol Med 36: 801-807, 2015.

4. Vernet D, Ferrini MG, Valente EG, Magee TR, Bou-Gharios G, Rajfer J and Gonzalez-Cadavid NF: Effect of nitric oxide on the differentiation of fibroblasts into myofibroblasts in the Peyronie's fibrotic plaque and in its rat model. Nitric Oxide 7: 262-276, 2002

5. Magee TR, Qian A, Rajfer J, Sander FC, Levine LA and Gonzalez-Cadavid NF: Gene expression profiles in the Peyronie's disease plaque. Urology 59: 451-457, 2002.

6. Zorba OU, Sirma S, Ozgon G, Salabas E, Ozbek U and Kadioglu A: Comparison of apoptotic gene expression profiles between Peyronie's disease plaque and tunica albuginea. Adv Clin Exp Med 21: 607-614, 2012.

7. Powell DW, Mifflin RC, Valentich JD, Crowe SE, Saada JI and West AB: Myofibroblasts. I. Paracrine cells important in health and disease. Am J Physiol 277: C1-C9, 1999.

8. Gelbard M: Myofibroblasts and mechanotransduction: Do forces in the tunica albuginea contribute to Peyronie's disease? J Sex Med 5: 2974-2976, 2008.

9. Arno AI, Amini-Nik S, Blit PH, Al-Shehab M, Belo C, Herer E and Jeschke MG: Effect of Human Wharton's Jelly Mesenchymal Stem Cell Paracrine Signaling on Keloid Fibroblasts. Stem Cells Transl Med 3: 299-307, 2014.

10. Kocher AA, Schlechta B, Gasparovicova A, Wolner E, Bonaros $\mathrm{N}$ and Laufer G: Stem cells and cardiac regeneration. Transpl Int 20: 731-746, 2007. 
11. Salibian AA, Widgerow AD, Abrouk M and Evans GR: Stem cells in plastic surgery: A review of current clinical and translational applications. Arch Plast Surg 40: 666-675, 2013.

12. Li L, Zhang S, Zhang Y, Yu B, Xu Y and Guan Z: Paracrine action mediate the antifibrotic effect of transplanted mesenchymal stem cells in a rat model of global heart failure. Mol Biol Rep 36: 725-731, 2009.

13. Iwamoto $\mathrm{T}$, Terai $\mathrm{S}$, Hisanaga $\mathrm{T}$, Takami $\mathrm{T}$, Yamamoto $\mathrm{N}$, Watanabe $\mathrm{S}$ and Sakaida I: Bone-marrow-derived cells cultured in serum-free medium reduce liver fibrosis and improve liver function in carbon-tetrachloride-treated cirrhotic mice. Cell Tissue Res 351: 487-495, 2013.

14. Gatti S, Bruno S, Deregibus MC, Sordi A, Cantaluppi V, Tetta C and Camussi G: Microvesicles derived from human adult mesenchymal stem cells protect against ischaemia-reperfusion-induced acute and chronic kidney injury. Nephrol Dial Transplant 26: 1474-1483, 2011.

15. Ortiz LA, Gambelli F, McBride C, Gaupp D, Baddoo M, Kaminski N and Phinney DG: Mesenchymal stem cell engraftment in lung is enhanced in response to bleomycin exposure and ameliorates its fibrotic effects. Proc Natl Acad Sci USA 100: 8407-8411, 2003.

16. Zhou Y, Yuan J, Zhou B, Lee AJ, Lee AJ, Ghawji M Jr and Yoo TJ: The therapeutic efficacy of human adipose tissue-derived mesenchymal stem cells on experimental autoimmune hearing loss in mice. Immunology 133: 133-140, 2011.

17. Tang WP, Akahoshi T, Piao JS, Narahara S, Murata M, Kawano T, Hamano N, Ikeda T and Hashizume M: Splenectomy enhances the therapeutic effect of adipose tissue-derived mesenchymal stem cell infusion on cirrhosis rats. Liver Int 36: 1151-1159, 2016.

18. Tzouvelekis A, Paspaliaris V, Koliakos G, Ntolios P, Bouros E, Oikonomou A, Zissimopoulos A, Boussios N, Dardzinski B, Gritzalis D, et al: A prospective, non-randomized, no placebo-controlled, phase Ib clinical trial to study the safety of the adipose derived stromal cells-stromal vascular fraction in idiopathic pulmonary fibrosis. J Transl Med 11: 171, 2013.

19. Burgos-Silva M, Semedo-Kuriki P, Donizetti-Oliveira C, Costa PB, Cenedeze MA, Hiyane MI, Pacheco-Silva A and Câmara NO: Adipose Tissue-Derived Stem Cells Reduce Acute and Chronic Kidney Damage in Mice. PLoS One 10: e0142183, 2015.

20. Yun IS, Jeon YR, Lee WJ, Lee JW, Rah DK, Tark KC and Lew DH: Effect of human adipose derived stem cells on scar formation and remodeling in a pig model: A Pilot Study. Dermatol Surg 38: 1678-1688, 2012.

21. Lam MT, Nauta A, Meyer NP, Wu JC and Longaker MT: Effective delivery of stem cells using an extracellular matrix patch results in increased cell survival and proliferation and reduced scarring in skin wound healing. Tissue Eng Part A 19: 738-747, 2013.

22. Lauer-Fields JL, Juska D and Fields GB: Matrix metalloproteinases and collagen catabolism. Biopolymers 66: 19-32, 2002.

23. Schofield AV and Bernard O: Rho-associated coiled-coil kinase (ROCK) signaling and disease. Crit Rev Biochem Mol Biol 48: 301-316, 2013.

24. Qiu X, Villalta J, Ferretti L, Fandel TM, Albersen M, Lin G, Dai Y, Lue TF and Lin CS: Effects of intravenous injection of adipose-derived stem cells in a rat model of radiation therapy-induced erectile dysfunction. J Sex Med 9: 1834-1841, 2012.

25. Zhu LL, Zhang Z, Jiang HS, Chen H, Chen Y and Dai YT: Superparamagnetic iron oxide nanoparticle targeting of adipose tissue-derived stem cells in diabetes-associated erectile dysfunction. Asian J Androl 19: 425-432, 2017.

26. Ahuja SK, Sikka SC and Hellstrom WJ: Stimulation of collagen production in an in vitro model for Peyronie's disease. Int J Impot Res 11: 207-212, 1999.

27. Yu FX, Su LF, Dai CL, Wang Y, Teng YY, Fu JH, Zhang QY and Tang YH: Inhibition of pancreatic stellate cell activity by adipose-derived stem cells. Hepatobiliary Pancreat Dis Int 14: 215-221, 2015.

28. Woessner JF Jr: The determination of hydroxyproline in tissue and protein samples containing small proportions of this imino acid. Arch Biochem Biophys 93: 440-447, 1961.

29. Sun Z, Wang C, Shi C, Sun F, Xu X, Qian W, Nie S and Han X: Activated Wnt signaling induces myofibroblast differentiation of mesenchymal stem cells, contributing to pulmonary fibrosis. Int J Mol Med 33: 1097-1109, 2014.

30. Xu F, Liu C, Zhou D and Zhang L: TGF- $/$ /SMAD Pathway and Its Regulation in Hepatic Fibrosis. J Histochem Cytochem 64: 157-167, 2016.
31. Li Y, Zhang W, Gao J, Liu J, Wang H, Li J, Yang X, He T, Guan H, Zheng Z, et al: Adipose tissue-derived stem cells suppress hypertrophic scar fibrosis via the p38/MAPK signaling pathway. Stem Cell Res Ther 7: 102, 2016.

32. Harn HJ, Lin SZ, Hung SH, Subeq YM, Li YS, Syu WS, Ding DC, Lee RP, Hsieh DK, Lin PC and Chiou TW: Adipose-derived stem cells can abrogate chemical-induced liver fibrosis and facilitate recovery of liver function. Cell Transplant 21: 2753-2764, 2012.

33. Zhang Q, Liu LN, Yong Q, Deng JC and Cao WG: Intralesional injection of adipose-derived stem cells reduces hypertrophic scarring in a rabbit ear model. Stem Cell Res Ther 6: 145, 2015.

34. Hattori $\mathrm{H}$ and Ishihara $\mathrm{M}$ : Altered protein secretions during interactions between adipose tissue- or bone marrow-derived stromal cells and inflammatory cells. Stem Cell Res Ther 6: 70, 2015.

35. Gagliano N, Arosio B, Grizzi F, Masson S, Tagliabue J, Dioguardi N, Vergani C and Annoni G: Reduced collagenolytic activity of matrix metalloproteinases and development of liver fibrosis in the aging rat. Mech Ageing Dev 123: 413-425, 2002.

36. Huang YR, Wei QX, Wan YG, Sun W, Mao ZM, Chen HL, Meng XJ, Shi XM, Tu Y and Zhu Q: Ureic clearance granule, alleviates renal dysfunction and tubulointerstitial fibrosis by promoting extracellular matrix degradation in renal failure rats, compared with enalapril. J Ethnopharmacol 155: 1541-1552, 2014.

37. García-Alvarez J, Ramirez R, Checa M, Nuttall RK, Sampieri CL, Edwards DR, Selman M and Pardo A: Tissue inhibitor of metalloproteinase-3 is up-regulated by transforming growth factor-beta 1 in vitro and expressed in fibroblastic foci in vivo in idiopathic pulmonary fibrosis. Exp Lung Res 32: 201-214, 2006.

38. Fang S, Xu C, Zhang Y, Xue C, Yang C, Bi H, Qian X, Wu M, $\mathrm{Ji} \mathrm{K}$, Zhao Y, et al: Umbilical cord-derived mesenchymal stem cell-derived exosomal MicroRNAs suppress myofibroblast differentiation by inhibiting the transforming growth factor-beta/SMAD2 pathway during wound healing. Stem Cells Transl Med 5: 1425-1439, 2016.

39. Thumkeo D, Watanabe $S$ and Narumiya S: Physiological roles of Rho and Rho effectors in mammals. Eur J Cell Biol 92: 303-315, 2013.

40. Fukata Y, Kimura K, Oshiro N, Saya H, Matsuura Y and Kaibuchi K: Association of the myosin-binding subunit of myosin phosphatase and moesin: Dual regulation of moesin phosphorylation by Rho-associated kinase and myosin phosphatase. J Cell Biol 141: 409-418, 1998.

41. Sopko NA, Hannan JL and Bivalacqua TJ: Understanding and targeting the Rho kinase pathway in erectile dysfunction. Nat Rev Urol 11: 622-628, 2014.

42. Kwon KD, Choi MJ, Park JM, Song KM, Kwon MH, Batbold D, Yin GN, Kim WJ, Ryu JK and Suh JK: Silencing histone deacetylase 2 using small hairpin RNA induces regression of fibrotic plaque in a rat model of Peyronie's disease. BJU Int 114: 926-936, 2014.

43. Gonzalez-Cadavid NF and Rajfer J: Mechanisms of disease: New insights into the cellular and molecular pathology of Peyronie's disease. Nat Clin Pract Urol 2: 291-297, 2005.

44. Golan-Gerstl R, Wallach-Dayan SB, Zisman P, Cardoso WV, Goldstein RH and Breuer R: Cellular FLICE-like inhibitory protein deviates myofibroblast fas-induced apoptosis toward proliferation during lung fibrosis. Am J Respir Cell Mol Biol 47: 271-279, 2012.

45. Rodgers KD, Rao V, Meehan DT, Fager N, Gotwals P, Ryan ST, Koteliansky V, Nemori R and Cosgrove D: Monocytes may promote myofibroblast accumulation and apoptosis in Alport renal fibrosis. Kidney Int 63: 1338-1355, 2003.

46. Horowitz JC, Rogers DS, Sharma V, Vittal R, White ES, Cui $\mathrm{Z}$ and Thannickal VJ: Combinatorial activation of FAK and AKT by transfonning growth factor-betal confers an anoikis-resistant phenotype to myofibroblasts. Cell Signal 19: 761-771, 2007.

47. Iekushi K, Taniyama Y, Azuma J, Sanada F, Kusunoki H, Yokoi T, Koibuchi N, Okayama K, Rakugi H and Morishita R: Hepatocyte growth factor attenuates renal fibrosis through TGF- $\beta 1$ suppression by apoptosis of myofibroblasts. J Hypertens 28: 2454-2461, 2010 . 